\title{
Port-Hamiltonian approaches to motion generation for mechanical systems
}

\author{
Satoru Sakai and Stefano Stramigioli
}

\begin{abstract}
This paper gives new motion generation methods for mechanical port-Hamiltonian systems. First, we propose a generation method based on an asymptotic stabilization method without damping assignment. This asymptotic stabilization method preserves the Hamiltonian structure in the closed-loop system although the controller itself is not a portHamiltonian system. Second, we propose another method based on an adaptive asymptotic stabilization method for unknown damping. This adaptive asymptotic stabilizer does not use the value and the sign of the damping at all. Finally, we confirm the effectiveness of our techniques in some numerical simulation.
\end{abstract}

\section{INTRODUCTION}

This paper gives new motion generation methods for portHamiltonian systems.

Hamiltonian control systems [10], [6] are systems described by Hamilton's equations which represent general physical systems. Recently port-controlled Hamiltonian systems are introduced as a generalization of Hamiltonian systems [4]. They can represent not only ordinary mechanical, electrical and electro-mechanical systems (e.g.: magnetic levitation system), but also a class of nonholonomic systems (e.g.: vehicle systems) [5], [3]. The special structure of physical systems allows us to utilize the passivity which they intrinsically possess and a lot of fruitful results were obtained so far. These methods are so called passivity based control [9], [7].

One of the advantages of passivity based control is robustness of the closed-loop system. It is usually difficult to stabilize a nonlinear system without using dynamic parameters, such as, mass and moment of inertia. Utilizing the intrinsic passive property of physical systems, however, it is easy to stabilize the system by using only the information of kinematic parameters. There are some design methods for port-Hamiltonian systems, generalized canonical transformation[2], IDA-PBC method, Casimir function method and so on. Especially, the canonical transformation approach can achieve not only stabilization but also tracking and dynamic output feedback stabilization [1], [8]. These transformations are natural generalization of the canonical transformations which are well-known in classical mechanics and preserve the structure of the port-controlled Hamiltonian systems.

S. Sakai is with Faculty of Electrical and Mechanical Engineering, Chiba University, Inage Yayoi 1-33, Japan satorusakai@faculty.chiba-u.jp

S. Stramigioli is with the Faculty of Electrical Engineering, University of Twente, $7500 \mathrm{AE}$ Enschede, The Netherlands
In this paper, we propose motion generation methods for port-Hamiltonian systems which is not studied yet in the comparison with the stabilization methods. We give two generation methods in this paper. The first one is based on an asymptotic stabilization method without damping assignment. This asymptotic stabilization method preserves the Hamiltonian structure in the closed-loop system although the controller itself is not a port-Hamiltonian system. The second one is based on an adaptive asymptotic stabilization method for unknown damping. This adaptive asymptotic stabilization method estimates not only the value of the damping but also the (maybe negative) sign of the damping.

This paper is organized as follows. In Section II, we review port-Hamiltonian systems and their existing stabilization methods. In Section III, we propose the first new asymptotic stabilization method and motion generation method without damping assignment. In Section IV, we give the adaptive asymptotic stabilization method and, in Section V, we propose the second new motion generation method based on the result of Section IV. In Section VI, we show the effectiveness of out techniques. In Section VII, we conclude this paper.

In this paper, a closed-loop system of two nonlinear systems:

$$
\Sigma_{i}:\left\{\begin{array}{l}
\dot{x_{i}}=f_{i}\left(x_{i}, u_{i}\right) \\
y_{i}=h_{i}\left(x_{i}, u_{i}\right), \quad i=p, c,
\end{array}\right.
$$

by connection $\left(u_{p}, u_{c}\right)=\left(y_{c},-y_{p}\right)$ is expressed as $\left\{\Sigma_{p}, \Sigma_{c}\right\}$. $\left[a_{i j}\right]$ is matrix $A$ whose $(i, j)$ components are $a_{i j},\|A\|_{F}$ is the Frobenius norm of $A, I_{n}$ is the identity matrix and $n$ can be omitted for its uniqueness and $\operatorname{vec}(A)$ is the vector formed by stacking the columns of $A$ into one long vector.

\section{Port-Hamiltonian Systems}

\section{A. Port-Hamiltonian systems [10]}

A port-Hamiltonian system with Hamiltonian $H(x, t)$ is expressed as

$$
\left\{\begin{array}{l}
\dot{x}=J(x, t) \frac{\partial H}{\partial x}(x, t)^{T}+g(x, t) u \\
y=g(x, t)^{T} \frac{\partial H}{\partial x}(x, t)^{T}
\end{array}\right.
$$

where, $u, y \in \mathbf{R}^{m}, x \in \mathbf{R}^{n} . J$ is a skew-symmetric matrix and

$$
J=\left[\begin{array}{ccc}
0 & I_{k} & 0 \\
-I_{k} & 0 & 0 \\
0 & 0 & 0
\end{array}\right]
$$


canonical coordinate $x=\left(q_{1}, \ldots, q_{k}, p_{1}, \ldots, p_{k}, s_{1}, \ldots, s_{l}\right)$. PortHamiltonian systems are a natural generalization of Hamiltonian systems

$$
\dot{x}=J \frac{\partial H^{T}}{\partial x}
$$

and the following properties are known.

Lemma 1 Consider the system (2). Suppose that the Hamiltonian $H$ is bounded from below and satisfies $\partial H / \partial t \leq 0$. Then the system is passive with respect to the storage function $H$, and the following feedback renders $(u, y) \rightarrow 0$. Furthermore, if the system is zero-state detectable, then the following feedback renders the system asymptotically stable

$$
\Sigma_{d}: u=-C y
$$

where $C>0$ is any positive definite matrix.

Remark 1 The zero-state detectability, which is assumed in Lemma 1, does not always hold for general systems. In such a case, the stabilization method by generalized canonical transformation (which is a generalization of a stabilization method of exploiting virtual potential energy [9]) is useful.

In this paper, we give two motion generation methods for port-Hamiltonian systems. The first one is based on an asymptotic stabilization method without damping assignment. The second one is based on an adaptive asymptotic stabilization method for unknown damping.

\section{Motion GENERATION WiThOUT DAMPING ASSIGNMENT}

\section{A. Asymptotic stabilization without damping assignment}

In the approach of Lemma 1, the closed-loop system does not preserve the Hamiltonian structure of (4), because the closed-loop system has the following form

$$
\dot{x}=(J-R) \frac{\partial H^{T}}{\partial x}
$$

with a positive definite matrix $R>0$.

Now, apart from this approach, we give an asymptotic stabilization method of port-Hamiltonian systems while preserving the Hamiltonian structure.

Theorem 1 Consider the following class of port-Hamiltonian systems (2).

$$
\Sigma_{m}:\left\{\begin{aligned}
{\left[\begin{array}{c}
\dot{q} \\
\dot{p}
\end{array}\right] } & =\left[\begin{array}{cc}
0 & I \\
-I & 0
\end{array}\right]\left[\begin{array}{l}
\frac{\partial H}{\partial q}^{T} \\
\frac{\partial H}{\partial p}^{T}
\end{array}\right]+\left[\begin{array}{l}
0 \\
G
\end{array}\right] u \\
y & =G^{T} \frac{\partial H}{\partial p}^{T}
\end{aligned}\right.
$$

where $G$ is nonsingular and $H$ is bounded from below.

Then, the input-output nulling set of $\Sigma, \Omega_{0} \equiv$ $\left\{\left(q^{T}, p^{T}\right)^{T} \mid u=y \equiv 0\right\}, \quad$ is asymptotically stabilized by the following controller.

$$
\Sigma_{c 1}:\left\{\begin{array}{l}
\dot{r}=-P_{r}^{T} G^{-T} y \\
u=G^{-1} P_{r} r
\end{array}\right.
$$

where,

$$
P_{r}=-C \frac{\partial H}{\partial p} r^{T}
$$

and $C>0$ is any positive definite matrix.

Proof of Theorem 1.

The positive definite function

$$
E_{r}=\frac{1}{2} r^{T} r
$$

has the following property:

$$
\begin{aligned}
\dot{E}_{r} & =r^{T} \dot{r} \\
& =r^{T}\left(-P_{r}^{T} \frac{\partial H^{T}}{\partial p}\right) \\
& =2 E_{r} \frac{\partial H}{\partial p} C \frac{\partial H^{T}}{\partial p} \geq 0 .
\end{aligned}
$$

On the other hand, the closed-loop system is given as

$$
\left[\begin{array}{c}
\dot{q} \\
\dot{p} \\
\dot{r}
\end{array}\right]=\left[\begin{array}{ccc}
0 & I & 0 \\
-I & 0 & P_{r} \\
0 & -P_{r}^{T} & 0
\end{array}\right]\left[\begin{array}{l}
\frac{\partial \bar{H}^{T}}{\partial q} \\
\frac{\partial \bar{H}^{T}}{\partial p} \\
\frac{\partial \bar{H}^{T}}{\partial r}
\end{array}\right]
$$

where $\bar{H}=H+E_{r}$ and

$$
\dot{\bar{H}}=0
$$

This means

$$
\dot{H}=\dot{\bar{H}}-\dot{E}_{r} \leq 0
$$

Since $H$ is bounded from below, the input-output nulling space $\Omega_{0}$, where $\dot{H} \equiv 0$, is asymptotically stable. (Q.E.D.)

This asymptotic stabilizer $\Sigma_{c 1}$ is not port-Hamiltonian systems in the sense of (2) and (3) and the minimal realization of energy controller in [11]. $P_{r}$ in the stabilizer requires the dynamic parameters which may have perturbations in practice. However, as far as the inequality (11) holds, we can use the result of Theorem 1 for a robust stabilization.

\section{B. Generation method without damping assignment}

By using the result of Theorem 1, we give a new motion generation method for port-Hamiltonian system.

First we will indicate with $T$ a map of a system $\Sigma_{c}$ like the ones in (1) to one of the same form and dimensions in the sense that the functions $f_{c}$ and $h_{c}$ will be respectively to functions of the same dimensions $T_{f}\left(f_{c}\right)$ and $T_{h}\left(h_{c}\right)$ respectively. Second, by using this reflection mapping, we give the new motion generation method without damping assignment.

Lemma 2 Consider $\Sigma_{p}$ which is dissipative with respect a storage function $S\left(x_{p}\right), \Sigma_{c}$ which is interconnected to $\Sigma_{p}$ by connection $\left(u_{p}, u_{c}\right)=\left(y_{c},-y_{p}\right)$. Suppose that $T \circ \Sigma_{c}$ satisfies

$$
\left(L_{\left\{\Sigma_{p}, \Sigma_{c}\right\}}+L_{\left\{\Sigma_{p}, T \circ \Sigma_{c}\right\}}\right) S\left(x_{p}\right)=0
$$

where $L_{\Sigma}$ is the Lie derivative along the trajectory on the vector field of the autonomous system $\Sigma$. Then the time 
derivative of $S$ along the trajectory of $\left\{\Sigma_{p}, T \circ \Sigma_{c}\right\}$ is given by the time derivative of $S$ along the trajectory of $\left\{\Sigma_{p}, \Sigma_{c}\right\}$ as

$$
\dot{S}\left(x_{p}\right) \stackrel{T}{\mapsto}-\dot{S}\left(x_{p}\right) .
$$

\section{Proof of Lemma 2.}

The poof is given by a straightforward calculation. (Q.E.D.)

Remark 2 In the case of $\Sigma_{p}$ is driftless systems $f_{p}=0$, or port-Hamiltonian systems (2), (16) is equivalent to the output ports sign reflection for $\Sigma_{c}$ as

$$
h_{c} \stackrel{T}{\mapsto}-h_{c} .
$$

Theorem 2 Consider the following class of port-Hamiltonian systems and let $H_{d}$ the desired Hamiltonian,

$$
\Sigma_{m}:\left\{\begin{array}{c}
{\left[\begin{array}{c}
\dot{q} \\
\dot{p}
\end{array}\right]=\left[\begin{array}{cc}
0 & I \\
-I & 0
\end{array}\right]\left[\begin{array}{l}
\frac{\partial H}{\partial q}{ }^{T} \\
\frac{\partial H}{\partial p}^{T}
\end{array}\right]+\left[\begin{array}{l}
0 \\
G
\end{array}\right] u} \\
y=G^{T} \frac{\partial H}{\partial p}^{T}
\end{array}\right.
$$

where $G$ is nonsingular and $H$ is bounded from below.

Then, a set $\Omega_{d}=\left\{\left(q^{T}, p^{T}\right)^{T} \mid H=H_{d}\right\}$ is asymptotically stabilized by the following controller

$$
T \circ \Sigma_{c 1}:\left\{\begin{array}{l}
\dot{r}=-P_{r}^{T} G^{-T} y \\
u=G^{-1} P_{r} r
\end{array}\right.
$$

where

$$
P_{r}=+C \frac{\partial H}{\partial p} r^{T}
$$

and $\|r(0)\|_{2}=2\left(H_{d}-H(0)\right)$ with $H_{d} \geq H(0)$.

\section{Proof of Theorem 2.}

By Thorem 1 and a calculation, it is shown that (20) satisfies (15) while $\left\{\Sigma_{p}, T \circ \Sigma_{c 1}\right\}$ has the Hamiltonian structure. (Q.E.D.)

It is obvious that the closed-loop system preserves Hamiltonian structure. For integrable $\Sigma_{m}, T \circ \Sigma_{c 1}$ generates a periodic motion. This motion can not be given by the usual damping controller. That is,

$$
T \circ \Sigma_{d}: u=+C y
$$

can not asymptotically stabilize any periodic motion.

For example, in the case of the periodic motion for space satellites, we may neglect the energy dissipation. However, there are many cases where we can not neglect it. In addition, we can not identify the energy dissipation exactly for practical mechanical systems.

Now, we start from an adaptive asymptotic stabilization for unknown energy dissipation.

\section{AdAPtive Stabilization}

Consider port-Hamiltonian systems with dissipation. In many cases, the sign of dissipation matrix is known and positive for natural and practical mechanical systems. However, the sign is not always positive for pre-compensated portHamiltonian systems. In this section, we give an adaptive asymptotic stabilization method for port-Hamiltonian systems with an unknown (and negative) dissipation.

Theorem 3 Consider the following class of port-Hamiltonian systems (2),

$$
\Sigma_{x}:\left\{\begin{array}{c}
{\left[\begin{array}{c}
\dot{q} \\
\dot{p}
\end{array}\right]=\left[\begin{array}{cc}
0 & I \\
-I & -\Theta
\end{array}\right]\left[\begin{array}{c}
\frac{\partial H}{\partial q}^{T} \\
\frac{\partial H}{\partial p}^{T}
\end{array}\right]+\left[\begin{array}{c}
0 \\
G
\end{array}\right] u} \\
y=G^{T} \frac{\partial H}{\partial p}^{T}
\end{array}\right.
$$

where $G$ is nonsingular, $H$ is bounded from below and $\Theta(=$ $\left.\left[\theta_{i j}\right]\right)$ is an unknown constant.

Then, the following controller

$$
\Sigma_{c 2}:\left\{\begin{array}{l}
\dot{\hat{\theta}}_{i j}=H \frac{\partial H}{\partial p_{i}} \frac{\partial H}{\partial p_{j}} \\
u=-G^{-1}\left(I_{n}-\hat{\Theta}\right) \frac{\partial H}{\partial p}{ }^{T}
\end{array}\right.
$$

makes $\Omega_{0}$ of $\Sigma_{x}$ asymptotic stable, where $\tilde{\Theta} \equiv \Theta-\hat{\Theta}$ and $\hat{\Theta}$ is the estimated value of $\Theta$.

Proof of Theorem 3.

Consider the following positive definite function

$$
V=H+\frac{1}{2}\|\tilde{\Theta}\|_{F}^{2} .
$$

The time derivative of $V$ is

$$
\begin{aligned}
\dot{V}= & \dot{H}+\Sigma_{i j}\left(\theta_{i j}-\hat{\theta}_{i j}\right)\left(-\dot{\hat{\theta}}_{i j}\right) \\
= & \left(\frac{\partial H}{\partial p}(-\Theta) \frac{\partial H^{T}}{\partial p}+\frac{\partial H}{\partial p} G u\right) \\
& +\Sigma_{i j}\left(\theta_{i j}-\hat{\theta}_{i j}\right)\left(-\dot{\hat{\theta}}_{i j}\right) \\
= & -\left(\frac{\partial H}{\partial p} \frac{\partial H^{T}}{\partial p}\right)
\end{aligned}
$$

and a semi-negative definite function. This implies that $\Omega_{0}$ is asymptotically stable. (Q.E.D.)

\section{ADAPTIVE MOTION GENERATION}

By generalizing result of Theorem 3, we give an adaptive motion generation for port-Hamiltonian systems with an unknown damping. In this case, the adaptive action is always required in the controller, even if the damping is positive as usual plants.

Theorem 4 Consider the following class of port-Hamiltonian systems and let $H_{d}$ the desired Hamiltonian,

$$
\Sigma_{x}:\left\{\begin{array}{c}
{\left[\begin{array}{c}
\dot{q} \\
\dot{p}
\end{array}\right]=\left[\begin{array}{cc}
0 & I \\
-I & -\Theta
\end{array}\right]\left[\begin{array}{c}
\frac{\partial H}{\partial q}^{T} \\
\frac{\partial H}{\partial p}^{T}
\end{array}\right]+\left[\begin{array}{c}
0 \\
G
\end{array}\right] u} \\
y=G^{T} \frac{\partial H}{\partial p}^{T}
\end{array}\right.
$$


where $G$ is nonsingular, $H$ is bounded from below, and $\Theta$ is an unknown constant.

Then, the following controller

$$
\Sigma_{c 3}:\left\{\begin{array}{l}
\dot{\hat{\theta}}_{i j}=\tilde{H} \frac{\partial H}{\partial p_{i}} \frac{\partial H}{\partial p_{j}} \\
u=-G^{-1}\left(c \tilde{H} I_{n}-\hat{\Theta}\right) \frac{\partial H}{\partial p}
\end{array}\right.
$$

makes the set $\Omega_{d}=\left\{\left(q^{T}, p^{T}\right)^{T} \mid H=H_{d}\right\}$ asymptotically stable, if

$$
\tilde{H}(0)^{2}+\|\tilde{\Theta}(0)\|_{F}^{2}<\left(1+c^{2}\right) H_{d}^{2}
$$

holds, where $c>0$ is gain, where $\tilde{\Theta} \equiv \Theta-\hat{\Theta}, \hat{\Theta}$ is the estimated value of $\Theta$ and $\tilde{H}=H-H_{d}$.

\section{Proof of Theorem 4.}

Let $r=\operatorname{vec}(\tilde{\Theta})$, then there exists a matrix $P$ such that $\dot{r}=$ $\tilde{H} P \frac{\partial H}{\partial p}^{T}$ is equivalent to the state equation of the controller. Now we describe the closed-loop system as follows:

$$
\begin{aligned}
& {\left[\begin{array}{c}
\dot{q} \\
\dot{p} \\
\dot{r}
\end{array}\right]=\left[\begin{array}{ccc}
0 & I & 0 \\
-I & -c \tilde{H} I+\tilde{\Theta} & 0 \\
0 & \tilde{H} P & 0
\end{array}\right]\left[\begin{array}{l}
\frac{\partial H}{\partial q}^{T} \\
\frac{\partial H}{\partial p}^{T} \\
\frac{\partial H}{\partial r}^{T}
\end{array}\right]} \\
& =\left[\begin{array}{ccc}
0 & I & 0 \\
-I & -c \tilde{H} I+\tilde{\Theta} & -\tilde{H} P^{T} \\
0 & \tilde{H} P & 0
\end{array}\right]\left[\begin{array}{l}
\frac{\partial H}{\partial q}^{T} \\
\frac{\partial H}{\partial p}^{T} \\
\frac{\partial H}{\partial r}^{T}
\end{array}\right] \\
& +\left[\begin{array}{c}
0 \\
\tilde{H} P^{T} \frac{\partial H}{\partial r}^{T} \\
0
\end{array}\right] \\
& =\left[\begin{array}{ccc}
0 & I & 0 \\
-I & -c \tilde{H} I+\tilde{\Theta}+\tilde{H} \tilde{\Theta} & -\tilde{H} P^{T} \\
0 & \tilde{H} P & 0
\end{array}\right]\left[\begin{array}{c}
\frac{\partial H}{\partial q}^{T} \\
\frac{\partial H}{\partial p}^{T} \\
\frac{\partial H}{\partial r}^{T}
\end{array}\right],
\end{aligned}
$$

where the structure of port-Hamiltonian system is still preserved. Then, we consider the following positive definite function

$$
V=\frac{1}{2}\left(\left(H-H_{d}\right)^{2}+\|\tilde{\Theta}\|_{F}^{2}\right)
$$

and its time derivative

$$
\begin{aligned}
\dot{V}= & \tilde{H} \dot{H}+\Sigma_{i j}\left(\theta_{i j}-\hat{\theta}_{i j}\right)\left(-\dot{\hat{\theta}}_{i j}\right) \\
= & \tilde{H}\left(\frac{\partial H}{\partial p}(-\Theta) \frac{\partial H^{T}}{\partial p}+\frac{\partial H}{\partial p} G u\right) \\
& +\Sigma_{i j}\left(\theta_{i j}-\hat{\theta}_{i j}\right)\left(-\dot{\hat{\theta}}_{i j}\right) \\
= & -c \tilde{H}^{2}\left(\frac{\partial H}{\partial p} \frac{\partial H^{T}}{\partial p}\right)
\end{aligned}
$$

which is a semi-negative definite function. This means that there are two equibulium sets, $\Omega_{d}$ and $\Omega_{0}$. We do not discuss the trivial case of $\Omega_{d}=\Omega_{0}$. Now we analyze the attraction region of $\Omega_{d}$.

We introduce two sets in the following. Because

$$
\dot{H}(q, p)=-\frac{\partial H}{\partial p}(c \tilde{H} I+\tilde{\Theta}) \frac{\partial H^{T}}{\partial p}>0
$$

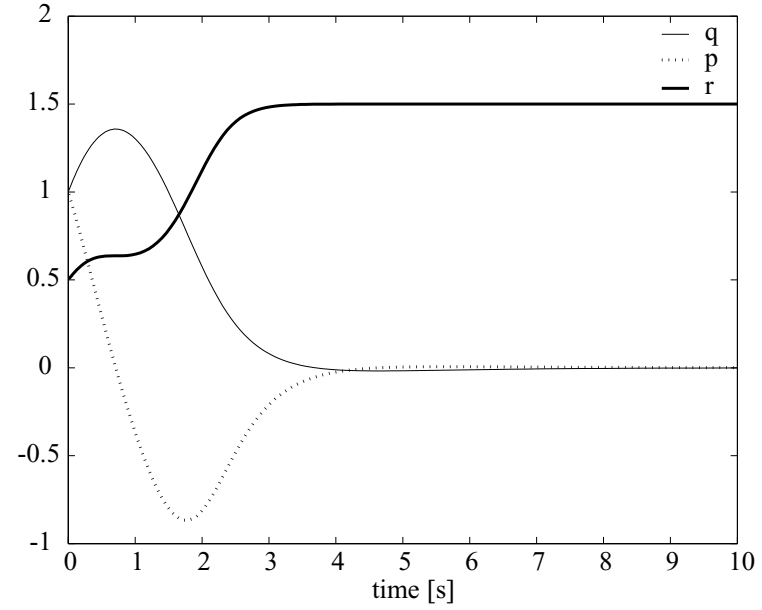

Fig. 1. Response of $q, p, r$

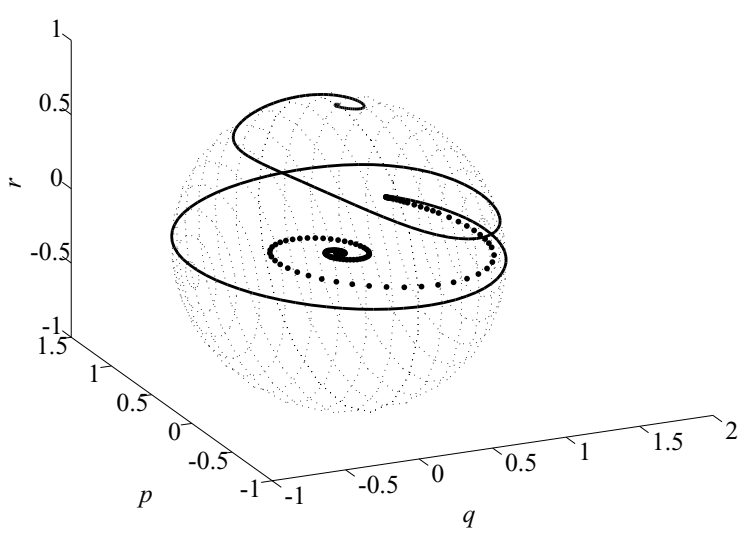

Fig. 2. Response in $(q, p, r)$ space

means $\tilde{\Theta}<-c\left(H-H_{d}\right) I, \Omega_{0 a s} \equiv\left\{(q, p) \mid \tilde{\Theta}<c H_{d} I\right\} \cap \Omega_{0}$ is the antistable subset of $\Omega_{0}$. Because of (30) and (31), $R\left(r_{b}\right) \equiv$ $\left\{(q, p) \mid V \leq r_{b}\right\}$ is positively invariant.

First, if $r_{b}$ is sufficiently small such that $R\left(r_{b}\right)$ does not contain any points in $\Omega_{0}$, that is, $R\left(r_{b}\right) \cap \Omega_{0}=\{0\}$, then $R(0)$ is asymptotically stable since (30) and (31) hold. Second, for larger $r_{b}$, even if $R\left(r_{b}\right) \cap \Omega_{0} \neq\{0\}$, as far as $\left(R\left(r_{b}\right) \cap\right.$ $\left.\Omega_{0}\right) \subset \Omega_{0 a s}$, then $R(0)$ is still asymptotically stable. Let $r_{b *} \equiv$ $r_{b}$ such that $\left(R\left(r_{b}\right) \cap \Omega_{0}\right) \subset \Omega_{0 a s}$. Every trajectory started from the positively invariant $R\left(r_{b *}\right)$ at except of $\Omega_{0}$ does not converse to $\Omega_{0}$ but converses to $R(0)$.

$r_{b *}$ can be maximized untill $\left(\partial\left(\Omega_{0}-\Omega_{0 a s}\right) \cap \partial R\left(r_{b *}\right)\right) \neq$ $\{0\}$, that is, $\tilde{\Theta}=c H_{d} I$ holds on $\partial R\left(r_{b *}\right)$ where $r_{b *}=$ $(1 / 2)\left(1+c^{2}\right) H_{d}^{2}$. This means that the attraction region of $R(0)$ is given by (28). (Q.E.D.)

In practice, the left hand of (28) is unknown and we design the gain $c$ by using the upper bounds of $\|\tilde{\Theta}(0)\|_{F}^{2}$.

Note that Theorem 4 is a generalization of Theorem 3 in the sense that the controller in (23) is a special case of that in (27). Furthermore, it is guaranteed that $V$ converses to 0 and $\tilde{\Theta}$ converses to 0 . 


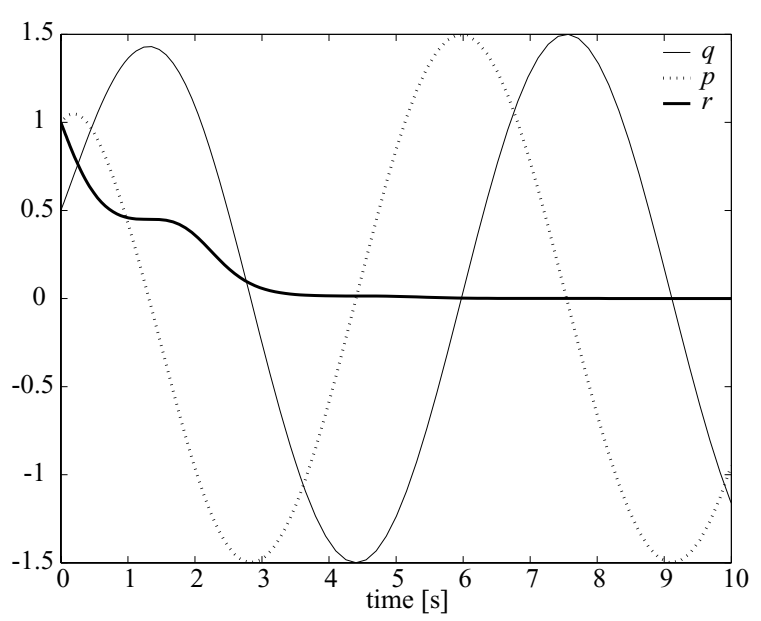

Fig. 3. Response of $q, p, r$

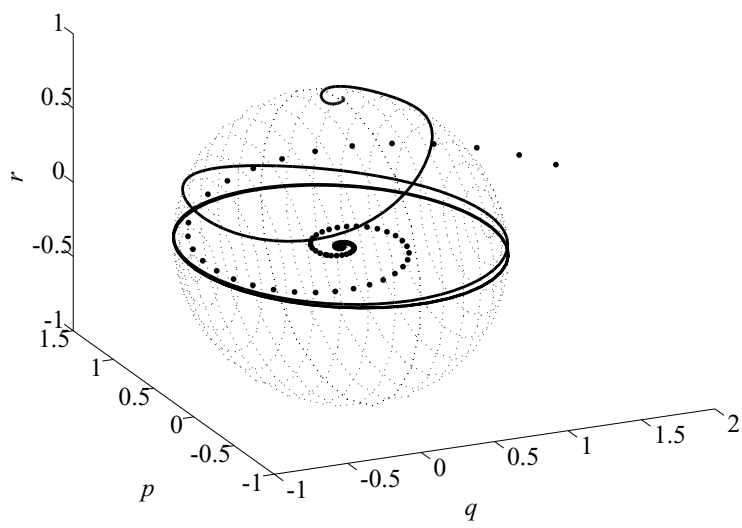

Fig. 4. Response in $(q, p, r)$ space

\section{NUMERICAL EXAMPLES}

Consider a 1-DOF mass-spring system. $q$ is the position of the mass and $p$ is the momentum. All parameters are normalized as 1 .

\section{A. Generation without damping assignment}

First we give a asymptotic controller by using Theorem 1. Initial values are $q(0)=1, p(0)=1$ and $r(0)=0.5$. Fig.1 shows responses of $q, p, r$. All states of plant $q, p$ are asymptotically stabilized to zero smoothly. The state of controller $r$ converses to $r(\infty)=\sqrt{q(0)^{2}+p(0)^{2}+r(0)^{2}}=$ 1.5 in its steady-state. The path of the closed-loop is given in Fig. 2 as the solid line. For comparison, we give a path by the existing damping controller (5) as the broken line. These results show the effectiveness of our method.

Then we generate a motion by using Theorem 2. Fig.3 shows the case of initial value $(q, p, r)=(1,1,0.5)$. As the magnitude of the controller state $r$ decreases, the plant state $(q, p)$ converse to a trajectory while only $p$ is used as output signal. The path of the closed-loop is given in Fig.4 as the solid line. For comparison, we give a path by the existing

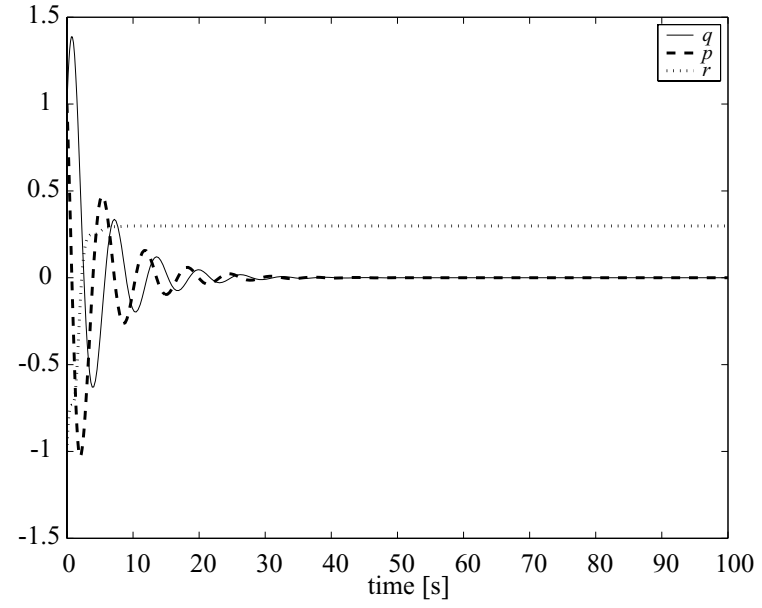

Fig. 5. Response of $q, p, r$

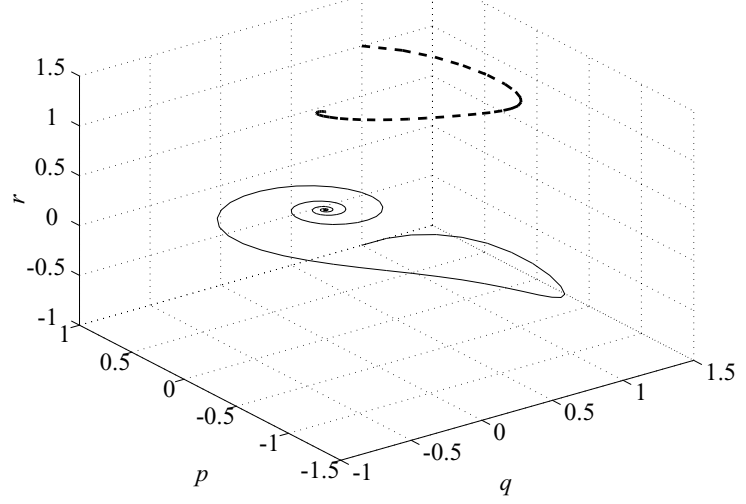

Fig. 6. Response of $q, p, r$

damping controller as the broken line. These results shows the effectiveness of our motion generation method.

\section{B. Generation with unknown damping}

First we derive the adaptive asymptotic stabilizer by using Theorem 3. The damping parameter is unknown and -1 . Initial values are $q(0)=1, p(0)=1$ and $r(0)=1$. Fig.5 shows the time response of the state $q, p, r$. The plant state $q, p$ converse to 0 smoothly and the controller state $r$ converses to a constant value. Fig. 6 shows the path of the closed-loop system as the solid line. For comparison, we show the case of the damping 1 as the broken line. It is confirmed that the origin of the plant is asymptotically stabilized for unknown negative and positive damping.

Second, we derive the adaptive generator by using Theorem 4. The desired Hamiltonian is $H_{d}=1.0$. Fig.7 shows the case of the initial value $(q, p, r)=(1,1,-1)$. As the controller state $r$ converses to 0 , the plant state $(q, p)$ converse to a trajectory though the damping parameter is unknown -1 . Fig. 8 shows the path of the closed-loop system. Since $r(0)$ is negative, there exists the overshoot. Fig.9 shows the case of initial value $(q, p, r)=(1,1,1)$. As the controller state $r$ converses to 0 , the plant state $(q, p)$ converse to the 


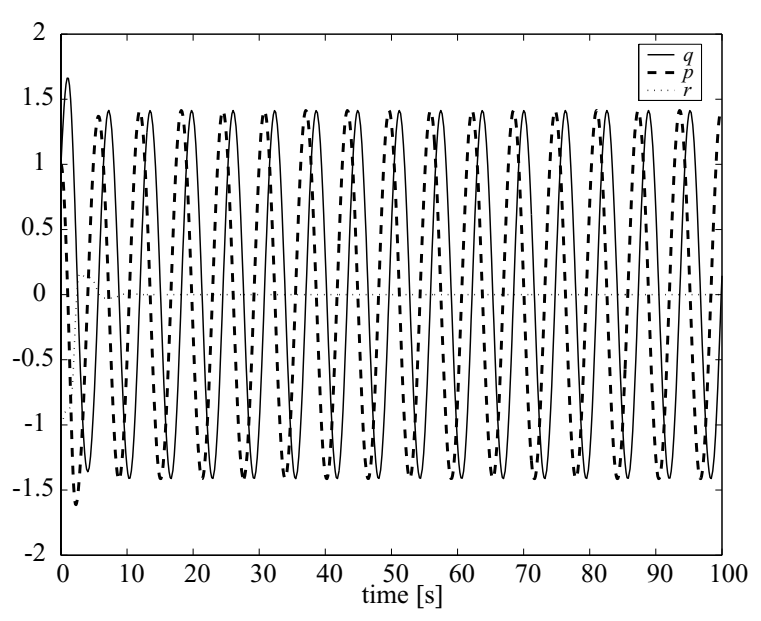

Fig. 7. Response of $q, p, r$

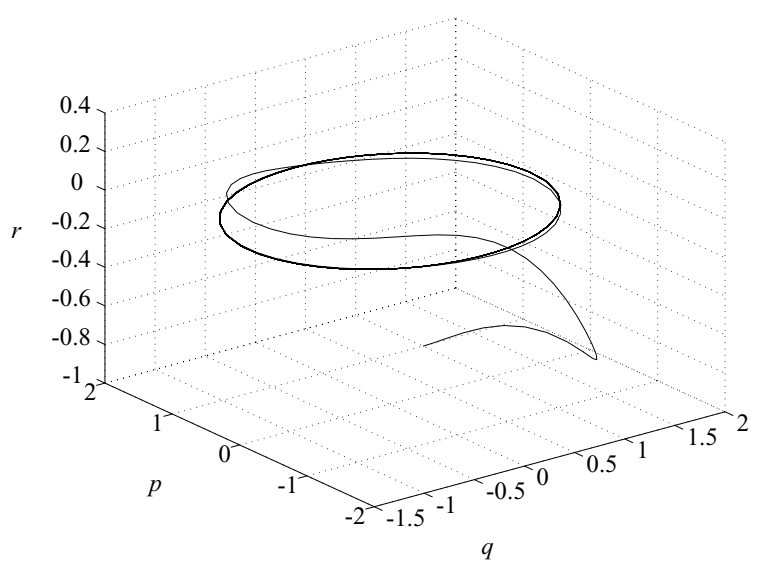

Fig. 8. Response in $(q, p, r)$ space

same trajectory, however the overshoot does not exist and $H$ decreases monotonically in Fig. 10 .

In all, the effectiveness of the proposed methods are confirmed.

\section{CONCLUSION}

We give two motion generation methods for portHamiltonian systems. The first one is based on an asymptotic stabilization method without damping assignment. The second one is based on an adaptive asymptotic stabilization method for unknown damping. Finally, we confirm the effectiveness of the proposed methods by numerical examples.

Further studies and applications to integrable systems are one of our future works.

Acknowledgment One of the authors would like to appreciate Prof. Kenji Fujimoto and Prof. Toshiharu Sugie.

\section{REFERENCES}

[1] K. Fujimoto, K. Sakurama, and T. Sugie. Trajectory tracking control of port-controlled Hamiltonian systems via generalized canonical transformations. Automatica, 39(12):2059-2069, 2003.

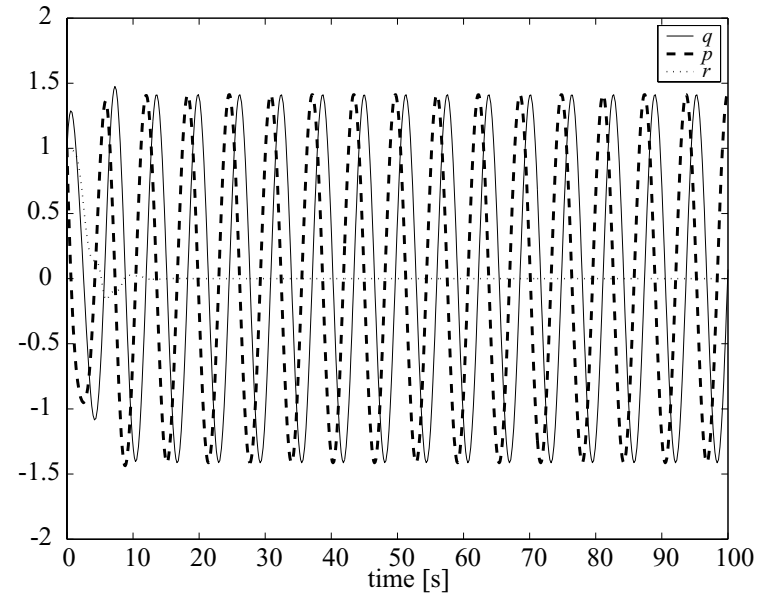

Fig. 9. Response of $q, p, r$

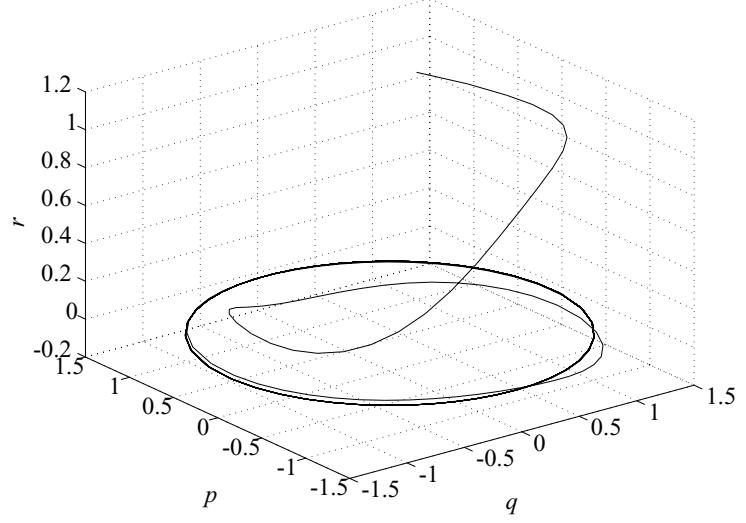

Fig. 10. Response in $(q, p, r)$ space

[2] K. Fujimoto and T. Sugie. Canonical transformation and stabilization of generalized hamiltonian systems. Systems \& Control Letters, 42(3):217-227, 2001.

[3] H. Khennouf, C. Canudas de Wit, A. J. van der Schaft, and J. Abraham. Preliminary results on asymptotic stabilization of Hamiltonian systems with nonholonomic constraints. In Proc. 34th IEEE Conf. on Decision and Control, pages 4305-4310, 1995.

[4] B. M. J. Maschke and A. J. van der Schaft. Port-controlled Hamiltonian systems: modeling origins and system-theoretic properties. In IFAC Symp. Nonlinear Control Systems, pages 282-288, 1992.

[5] B. M. J. Maschke and A. J. van der Schaft. A Hamiltonian approach to stabilization of nonholonomic mechanical systems. In Proc. 33rd IEEE Conf. on Decision and Control, pages 2950-2954, 1994.

[6] H. Nijmeijer and A. J. van der Schaft. Nonlinear Dynamical Control Systems. Springer-Verlag, New York, 1990.

[7] R. Ortega, A. Loria, P. J. Nicklasson, and H. Sira-Ramirez. Passivitybased Control of Euler-Lagrange Systems. Springer-Verlag, London, 1998.

[8] S. Sakai and K. Fujimoto. Dynamic output feedback stabilization of a class of nonholonomic hamiltonian systems. In Proc. IFAC World Congress 2005, pages 1967-1970, 2005.

[9] M. Takegaki and S. Arimoto. A new feedback method for dynamic control of manipulators. Trans. ASME, J. Dyn. Syst., Meas., Control, 103:119-125, 1981.

[10] A. J. van der Schaft. Stabilization of Hamiltonian systems. Nonl. An. Th. Math. Appl., 10:1021-1035, 1986.

[11] Arjan van. der. Schaft. Theory of port-hamiltonian systems. In Network modeling and control of physical systems, pages DISC, manuscript, 2005. 